\title{
Efeitos do trabalho em turnos nos controladores de tráfego aéreo: uma revisão sistemática baseada no método PRISMA
}

\author{
Alisson Vieira Marcolino ${ }^{a}$, Joseana Celiza Fernandes Siqueira ${ }^{b}$, \\ Bárbara Iansá de Lima Barroso ${ }^{a}$ \\ aDepartamento de Terapia Ocupacional, Universidade Federal da Paraíba - UFPB, João Pessoa, PB, Brasil. \\ bPrograma de Pós-graduação em Engenharia de Produção, Universidade Federal da Paraíba - UFPB, \\ João Pessoa, PB, Brasil.
}

\begin{abstract}
Resumo: Os controladores de tráfego aéreo (CTA) desempenham funções bastante complexas e de grande responsabilidade. Devido às necessidades do trabalho, eles são submetidos a regime de turnos que tornam seus horários irregulares. A alternância dos turnos pode causar efeitos que acarretam prejuízos no desempenho da função laboral e na qualidade de vida. Objetivo: explorar os efeitos que o trabalho em turnos causa nos CTA através de uma revisão sistemática no modelo PRISMA. Procedimentos metodológicos: A revisão sistemática foi realizada a partir de três bases de dados (PubMed, Science Direct e Web Of Science). Resultados: foram encontrados 748 artigos, sendo 487 da PubMed, 240 da Science Direct e 21 da Web Of Science, após a aplicação dos critérios de elegibilidade foram incluídos 7 artigos na revisão. Conclusões: os estudos mostraram efeitos que afetam principalmente os turnos da noite, causados por alterações na qualidade do sono, sonolência e fadiga, que acarretam queda no desempenho e aumentam os riscos de acidentes aéreos.
\end{abstract}

Palavras-chave: Trabalho em Turnos, Saúde do Trabalhador, Condições de Trabalho, Terapia Ocupacional.

\section{Effects of shift work in air traffic controllers: a systematic review based on the Prisma method}

\begin{abstract}
Air Traffic Controllers (ATC) perform very complex functions of great responsibility. Due to the necessities of the job, they are submitted to a work shift system which makes their schedule irregular. The alternation of work shifts can result in effects that cause damages to their work performance and quality of life. Objective: To explore the effects of shift work in ATCs through a systematic review based on the Preferred Reporting Items for Systematic Reviews and Meta-Analysis model (PRISMA). Methodological procedures: The systematic review was conducted based on three databases: PubMed, Science Direct and Web of Science. Results: A total of 748 articles were found, 487 from PubMed, 240 from Science Direct and 21 from Web of Science; seven articles were included in the review after the application of eligibility criteria. Conclusions: The researches showed effects that affect mainly the night shifts, caused by alteration in sleep quality, somnolence and fatigue, resulting in decreased performance and increasing the risk of air accidents.
\end{abstract}

Keywords: Shift Work, Worker Health, Work Conditions, Occupational Therapy.

\footnotetext{
Autor para correspondência: Bárbara Iansã de Lima Barrosoa, Centro de Ciências da Saúde, Departamento de Terapia Ocupacional, Universidade Federal da Paraíba, $2^{\circ}$ andar LASTE, Campus Universitário I, Cidade Universitária, CEP: 58059-900, João Pessoa, PB, Brasil, e-mail: barbarabarroso@yahoo.com.br
}

Recebido em Ago. 4, 2014; $1^{\text {a }}$ Revisão em Out. 30, 2014; Aceito em Nov. 18, 2014. 


\section{Introdução}

Ao longo de sua vida, o homem se engaja em diversas atividades, dentre elas a profissional, que pode causar interferências positivas e/ou negativas. O trabalho tem seu papel estruturante para o ser humano, e pode causar influências nos âmbitos físico e mental. Tanto os aspectos das condiçóes quanto os da organização do trabalho podem afetar a saúde dos trabalhadores (ALENCAR; BIZ, 2012).

Compreender as dimensôes do trabalho não é tarefa fácil. Seus efeitos refletem-se nas relações sobre a saúde do ser humano, merecendo uma maior atenção e uma exploração através de estudos clínicos que partam de situaçóes concretas de trabalho (LANCMAN; GHIRARDI, 2002). Refletir acerca das questôes que compreendem a natureza dos fatores psicossociais é complexo, abrangendo questóes associadas aos trabalhadores, meio ambiente geral e do trabalho (FISCHER, 2012).

A reflexão sobre a atividade profissional tem grande importância para a percepção e intervenção em situaçôes que predisponham ao surgimento de agravos à saúde e à diminuição do desempenho. Compreender a relação existente entre o trabalhador e seu labor faz parte da essência primária da terapia ocupacional, a qual visa investigar o problema apresentado ao tratar o sujeito para que ele reconheça seus limites e se estruture quanto aos fatores estressores internos e externos, de modo a transformar as situações de trabalho ao seu redor (LANCMAN, 2004).

As atividades do Controlador de Tráfego Aéreo (CTA) surgiram a partir das necessidades de auxílio aos pilotos a fim de permitirem uma maior segurança durante a navegaçáo como forma de prevenir colisóes entre aeronaves; tais atividades tornaram-se fundamentais após a II Guerra Mundial, quando houve um crescimento significativo da aviação com passageiros civis. Sua função é garantir segurança na fluência do tráfego aéreo, regularidade e eficácia, tudo através do processamento de informaçóes (BRASIL, 2014; MIGUEL et al., 2006).

Os CTA são responsáveis por controlar o tráfego de aeronaves, o qual se consubstancia mediante a orientação e aplicação dos planos de voos, além de providenciar socorros, autorizar manobras das aeronaves e garantir o cumprimento das normas e regulamentos determinados pelas autoridades aeronáuticas (BRASIL, 2014; CARVALHO; FERREIRA, 2012).

Informações como condições meteorológicas imperantes e as limitaçôes operacionais da aeronave são recebidas através de equipamentos diversos, e então analisadas e avaliadas pelo controlador. De acordo com a avaliação realizada durante o trabalho, ele deverá tomar decisóes rápidas sobre a necessidade de intervenção ou divulgação das informações. (EDWARDS et al., 2012; KIRCHNER; LAURIG, 1971; MOTTER; GONTIJO, 2007).

As atividades de controle de tráfego aéreo estão distribuídas entre o controle de radar que envolvem o controle de área e aproximaçáo e controle visual, basicamente nas torres de controle dos aeroportos (BISSERET, 1971; BRASIL, 2014; CARVALHO; FERREIRA, 2012; DEPARTAMENTO DE CONTROLE DO ESPAÇO AÉREO, 2014; KIRCHNER; LAURIG, 1971; KUO; JOU; LIN, 2012; SOARES; PINHEIRO; SOUSA, 2011). Dadas as necessidades da aviação mundial, esses serviços necessitam funcionar em jornadas ininterruptas de 24 horas por dia, 7 dias na semana (MIGUEL et al., 2006), o que torna inevitável o trabalho em turnos ou em horários irregulares.

O sistema de trabalho em turnos pode ser caracterizado de forma fixa ou rotativa, em regime de escala ou plantão; ele é definido como sendo a continuidade da produção ou prestação de serviços realizados em horários diurnos ou não, com ou sem interrupção diária, durante os dias úteis ou nos 7 dias da semana (SILVA, 2008).

Durante grande parte de sua vida, o trabalhador em turnos está na contramão da sociedade diurna, não apenas durante as jornadas noturnas, mas também nos horários vespertinos, fins de semana e feriados (FISCHER; LIEBER, 2003).

De acordo com Araújo (2000), a falta de regularidade dos horários de trabalho gera inconstância nas tarefas domésticas, familiares e sociais. No período de repouso e de recreação, representam consequências negativas relacionadas a essa forma de organizaçáo ocupacional. Ainda segundo o autor, esse tipo de sistema favorece o processo saúde-doença, posto que acarreta problemas de saúde e perturbaçôes sociofamiliares.

Turnos longos, horas extras e turnos noturnos causam diversos efeitos, entre eles a redução no desempenho das atividades, desconforto e fadiga física (CARUSO et al., 2004). Diversos tipos de doenças e as perturbações no ciclo circadiano que causam distúrbios do sono, estáo entre os problemas de saúde originados pelo trabalho (ARAÚJO, 2000; CARUSO et al., 2004; FLO et al., 2012; FROST; KOLSTAD; BONDE, 2009; KOSTREVA; MCNELIS; CLEMENS, 2002; NOONE, 2009; PAN et al., 2011; SIGNAL; GANDER, 2007). 
As perturbaçóes no ciclo circadiano, associados ao estresse do trabalho do CTA, geram redução do desempenho físico e ocupacional, o que pode estar associado à diminuição da atenção e da agilidade, essenciais para prevenção de acidentes aéreos (FOLKARD; CONDOR, 1987).

Conforme Jou, Kuo e Tang (2013), 90\% dos eventos relacionados à segurança aérea em Taiwan no período de 2000 a 2009 foram devidos a erros humanos, seguidos por fatores ambientais e problemas mecânicos da aeronave. Esses autores citam dificuldades na interação entre piloto e controlador de tráfego aéreo como a causa mais comum para colocar o voo em risco.

Devido aos CTA serem submetidos diariamente a tarefas bastante complexas que exigem um alto nível de atenção para atender às rápidas demandas decorrentes do seu trabalho, exigindo baixa tolerância a erros e desempenhadas muitas vezes em horários irregulares, é necessário conhecer as características do trabalho em turnos e seus efeitos, para possíveis intervençôes que previnam agravos à saúde e promovam um melhor desempenho e qualidade de vida a estes profissionais.

Portanto, iniciar uma reflexão a partir de uma revisão sistemática, em pesquisas publicadas relacionadas ao tema, é de grande relevância para a obtenção do conhecimento da realidade do trabalho em turnos e como este pode interferir no desempenho e na qualidade de vida dos CTA.

Para isso, esta revisão se baseou no modelo Preferred Reporting Items for Systematic Reviews and Meta-Analyses (PRISMA), que consiste em uma lista de verificação contendo 27 itens e um diagrama de fluxo de quatro fases. A lista inclui itens considerados essenciais para a comunicação transparente de uma revisão sistemática (LIBERATI et al., 2009).

Essa ferramenta permite que os autores realizem um check list dos itens que compóem os dados antes da submissão dos artigos de revisão sistemática, bem como auxilia os pesquisadores durante a realizaçáo do processo de revisão (PÁDULA et al., 2012).

Dessa forma, o objetivo deste estudo foi explorar os efeitos que o trabalho em turnos podem causar nos controladores de tráfego aéreo através de uma revisão sistemática, utilizando o método PRISMA. Como forma de compreender quais os tipos de trabalho em turnos aplicados aos controladores de tráfego aéreo; analisar a melhor forma de aplicabilidade da jornada de trabalho e como os efeitos causados pelos turnos influenciam na atividade profissional.

\section{Procedimentos metodológicos}

A formulação da pergunta e estratégia utilizada para compor este estudo baseou-se no modelo Population, Intervention Comparison, Outcome (PICO), em português "População, Intervenção, Comparação, Desfecho”. Esse modelo é bastante utilizado na Prática Baseada em Evidências (PBE) e recomendado para revisóes sistemáticas (SANTOS et al., 2007).

\subsection{Estratégia de busca}

A estratégia utilizada compreendeu a efetivação de buscas sistemáticas na literatura, a partir das bases de dados PubMed, Science Direct e Web OfScience. As buscas foram realizadas por artigos disponíveis até o dia 9 de junho de 2014. Não foram utilizados filtros de data, pois pretendeu-se realizar uma revisão de todos os estudos publicados até a atualidade.

Durante a revisão sistemática, foram utilizadas palavras incluídas nos recursos dos Descritores em Ciências da Saúde (DeCS) e alguns Termos Livres que não foram encontrados no DeCS nem no Medical Subject Headings (MeSH). Ainda foram inseridas algumas variaçóes, as quais resultaram na seguinte combinação: "air traffic control" OR "air traffic controller" $O R$ "air traffic controllers" $O R$ "flight control" OR "flight controller" OR "flight controllers" AND shiftwork OR "shift work". Tais combinaçôes e variaçóes dos descritores foram utilizadas como forma de ampliar as possibilidades de encontrar resultados significativos referentes ao tema. Os descritores foram escritos na língua inglesa, como forma de ampliar o potencial de busca das publicações.

\subsection{Critérios de elegibilidade}

Durante a revisão sistemática, foram considerados elegíveis os estudos 1) realizados com controladores de tráfego aéreo de ambos os sexos em qualquer faixa etária; 2) que abordam o trabalho em turnos; 3) publicados nos idiomas português, inglês ou espanhol; 4) disponíveis através de texto completo; 5) acessíveis na rede da Universidade Federal da Paraíba para download gratuito.

\subsection{Seleção dos artigos}

Os artigos foram baixados em todas as bases de dados escolhidas através do navegador Firefox UFPB e também na rede de computadores da própria instituição. 
Os arquivos que continham as referências extraídas das bases de dados foram adicionados no software Mendley Desktop para verificação e identificação de duplicidade.

Após esse procedimento, os artigos foram avaliados hierarquicamente pelo título, resumo e, finalmente, procedeu-se a leitura integral deles, para que fossem aplicados os critérios de elegibilidade predefinidos para a inclusão e a exclusão dos estudos.

\subsection{Extração dos dados}

Após o cumprimento das etapas anteriores, um revisor realizou a extraçáo dos seguintes dados: autoria; ano de publicação; características das amostras; escalas de turnos de trabalho; efeitos que o trabalho pode causar em turnos e os desfechos de cada estudo.

\section{Resultados}

Com o término das buscas nas bases de dados através dos descritores anteriormente mencionados, foram encontrados 748 artigos, sendo 487 na PubMed, 240 na Science Direct e 21 na Web Of Science.

Conforme a aplicaçáo dos critérios de elegibilidade para a inclusão e exclusão dos estudos, foram excluídos 516 estudos pelo título e 216 trabalhos pelo resumo (abstract). Os motivos mais comuns para exclusão nesta etapa foram estudos aplicados com outros profissionais, principalmente em enfermeiros e trabalhadores de linhas produtivas fabris. Também foram encontrados e excluídos estudos de revisão sistemática, meta-análises, sem o texto completo disponível e com abstract não disponível.

Os estudos selecionados em todas as bases para revisão do texto completo passaram por uma verificação de duplicidade que resultou na exclusão de 6 , posteriormente mais 3 foram excluídos por não obterem dados relevantes para essa revisão. Após todo o processo de seleção e aplicação dos critérios de elegibilidade, foram incluídos 7 artigos nesta revisão sistemática. Todo esse processo de seleção e aplicação dos critérios de elegibilidade pode ser observado através do fluxograma do modelo Prisma na Figura 1.

Para facilitar a visualização dos dados extraídos para análise, utilizou-se um quadro com as seguintes variáveis: autor, ano, amostra, escala de trabalho e desfechos, que podem ser observados através da Tabela 1.

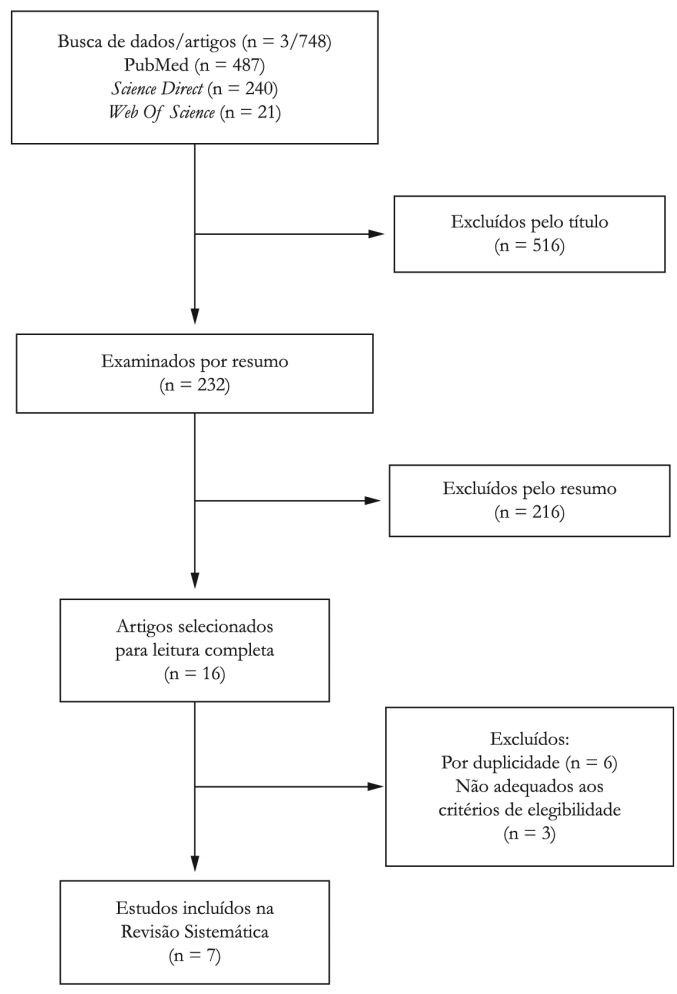

Figura 1. Fluxograma baseado no modelo PRISMA com os resultados da seleção dos artigos.

\section{Discussão}

Os estudos incluídos nesta revisão sistemática mostraram que existem diversos efeitos causados pelo trabalho em turnos com CTA, principalmente nos horários noturnos. Entre os efeitos imediatos, 4 artigos falam sobre a sensação de fadiga, outros 3 destacam a má qualidade do sono, sempre associando à diminuição da vigilância e do estado de alerta nos horários de trabalho. Três artigos integraram alguns desses efeitos ao ritmo circadiano, que sofre alteraçóes devido aos horários irregulares. Alguns estudos ainda relataram alteraçôes de humor e sintomas de estresse. Essas constataçôes - combinadas com as condiçóes de organização de trabalho e como ele se estrutura - afetam a qualidade de vida e, por conseguinte, a saúde do profissional aeroviário.

A alternância entre os turnos causa perturbaçôes qualitativas no sono, e o turno da noite é o mais prejudicado com os efeitos das rotaçóes. Testes realizados por Schroeder, Rosa e Witt (1988) mostraram que esse horário contribuiu para que houvesse tempos de respostas mais lentos e aumento nos erros, em relação aos outros horários. Esses efeitos podem ser explicados pela influência que as variadas direçôes de mudanças nos turnos causam 


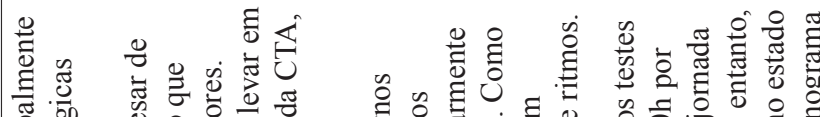

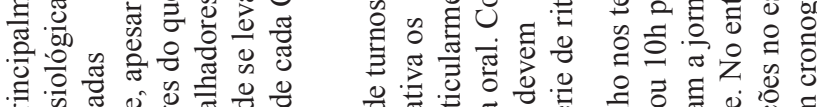

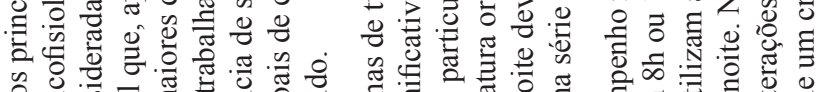

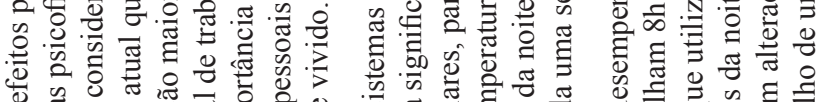

ป

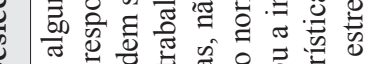

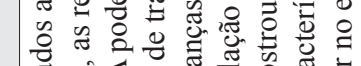

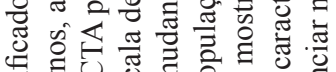

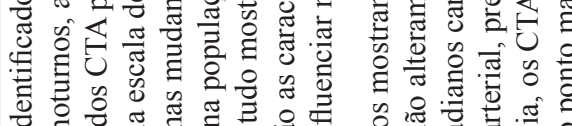

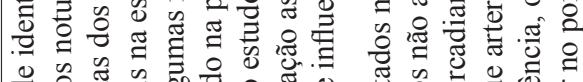

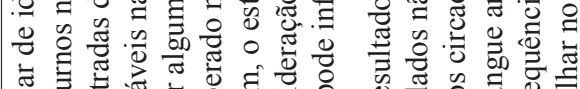

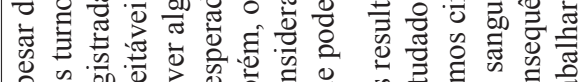

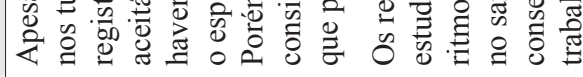

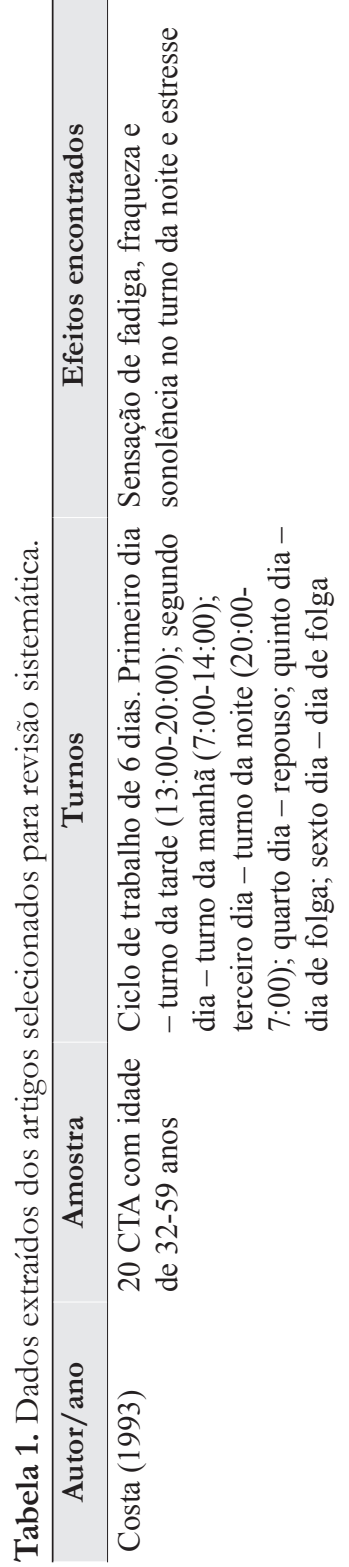

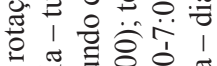

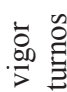

용

胥

的.

究

तु

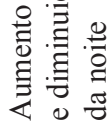

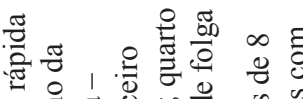

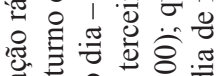

苨

ठำ ते

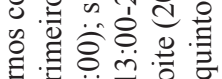

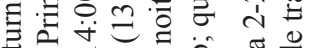

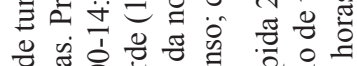
ช :

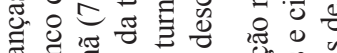

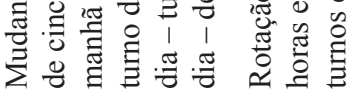

- 0 을

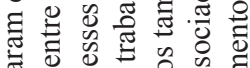

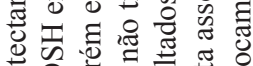

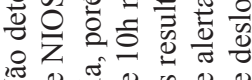

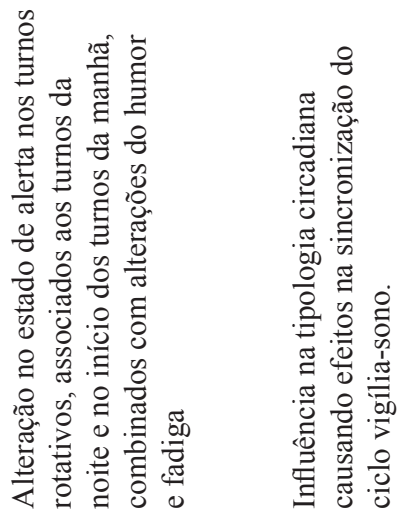

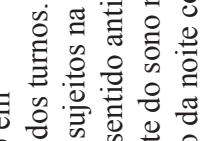
ब 章.

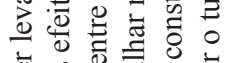
离

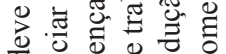
造离

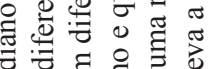
एँ 웡

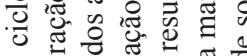
\&

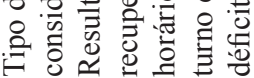




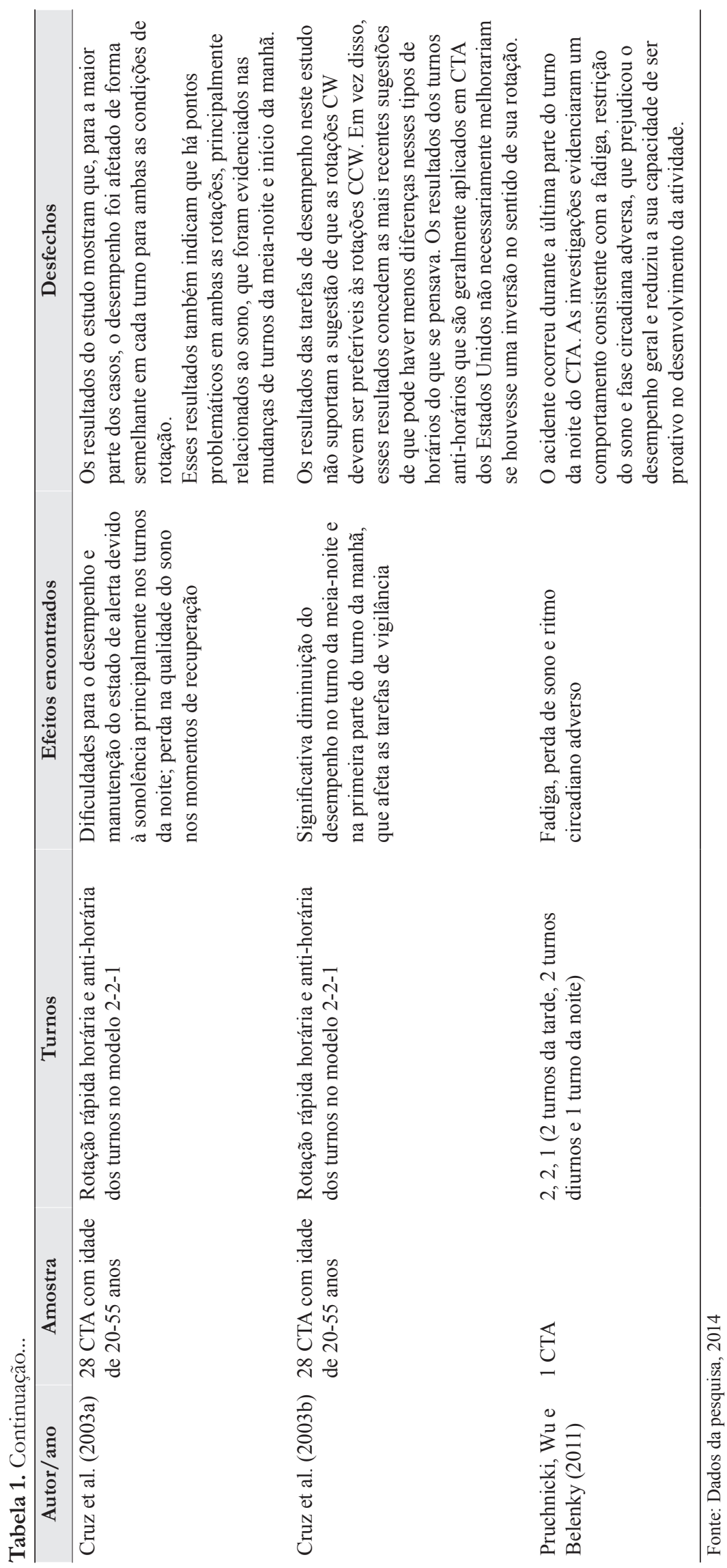


no ritmo circadiano, que é determinado por eventos internos e externos e tem ciclos de aproximadamente 24 horas, fato que determina os padróes temporais de cada espécie (SOUZA et al., 2012).

As alteraçóes no ritmo circadiano corroboram com efeitos que interferem negativamente no CTA; segundo estudo realizado por Folkard e Condon (1987), isso gera reduçáo do desempenho, que pode estar associado à diminuição da atenção e da agilidade, essenciais para a execução da função do profissional responsável pelo controle do tráfego aéreo. Essas condiçôes póem em risco a segurança aérea, posto que aumenta a probabilidade de erro humano na tomada de decisão, análise dos dados ou comunicação, que são causas de acidentes.

Foram encontradas diversas formas de rodízio dos turnos, horários que se direcionam no sentido horário e anti-horário, permitindo turnos nos horários matutinos, vespertinos e noturnos, combinados com dias de descanso e folga.

O tipo de alternância mais frequente encontrado foi a 2-2-1; nela, o CTA trabalha em um ciclo de 2 dias à tarde, 2 dias pela manhã e 1 dia à noite. Também foram encontradas escalas com ciclos de 6 dias de turnos (1 à tarde, 1 na manhă, 1 à noite, seguidos de 1 dia de repouso e 2 dias de folga), e o ciclo de 5 dias de turnos, iniciando com 1 turno pela manhã, 1 turno à tarde e 1 turno à noite, acrescidos de 1 dia de descanso e mais 1 dia de folga.

Nas escalas com rotaçóes 2-2-1, a sonolência no turno da noite é evidente. Isso pode ser explicado, pois o turno noturno inicia imediatamente após o último turno da manhâ, configurando uma rotação curta, já que o intervalo de horas é menor e acontece durante o dia, dificultando a recuperaçáo do sono. Essas atividades diárias acabam impedindo que o indivíduo recupere o sono, deixando para cochilar apenas momentos antes do início do turno da noite. Alguns CTA - principalmente os mais jovens - ainda preferem esse tipo de alternância de turnos, devido à necessidade de trabalhar em apenas um horário noturno e maior quantidade de horas de folga.

O ciclo de 6 dias na escala de trabalho também apresenta influências negativas no turno da noite, mas a adoção de uma rápida mudança no sistema de rotação com a inclusão de 2 dias de descanso após o turno da noite e a possibilidade de dormir um pouco durante o horário do turno mostram-se favoráveis, pois facilitam a adaptação psicofisiológica e compensam o déficit de sono, evitando queda de desempenho do CTA.

No ciclo de 5 dias, o estudo encontrou que, no sistema de avanço rápido com pausas programadas, não se alteram os ritmos circadianos cardiovasculares e não se induz uma dessincronização entre a pressão arterial e a temperatura oral. Porém os CTA que trabalham no turno da noite exercem suas funçôes no ponto mais baixo de uma série de ritmos, aumentando a fadiga e causando reduçáo no vigor e na vigilância.

O trabalho com alternância dos turnos não só causa efeitos no desempenho durante a jornada de trabalho, de modo que se pode perceber que, em todas as escalas de trabalho aplicadas aos CTA, há uma falta de regularidade dos horários de trabalho, o que gera inconstância nas tarefas domésticas e familiares. De acordo com Araújo (2000), isso representa consequências negativas relacionadas a essa forma de organização de trabalho, favorecendo problemas de saúde e perturbaçóes sociofamiliares.

A vida humana se compóe de um conjunto de situaçôes e que as atividades extralaborais são essenciais à qualidade de vida (EMMEL, 2012). Os estudos de Castro, Lima e Brunello (2001) abordam a qualidade de vida envolvendo a compreensão subjetiva das pessoas sobre seu bem-estar e sua situação de vida. Cada indivíduo tem sua singularidade no engajamento em suas atividades cotidianas fora do trabalho. Com isso o trabalho em turnos pode causar ou sofrer influências inerentes às atividades extralaborais, como: atividades de vida diária, atividades instrumentais de vida diária, lazer e participaçáo social, essenciais para o contexto de estudo e intervenção do terapeuta ocupacional.

A compreensão da inter-relação dessas esferas ligadas à identidade do sujeito faz parte das prerrogativas do terapeuta ocupacional, que considera a importância do trabalho e sua influência em todos os âmbitos do viver, utilizando-se de uma abordagem mais preventiva, buscando compreender a centralidade do trabalho na organização social e psíquica, como também na determinação do bem-estar dentro e fora do ambiente de trabalho (LANCMAN; GHIRARDI, 2002).

\section{Considerações finais}

Os estudos sobre o trabalho em turnos nos CTA ainda são pouco abordados pelos pesquisadores, principalmente quando se relacionam aos efeitos que esse tipo de escala de horários causa no desempenho e na saúde do profissional.

Foi identificado que os CTA que se submetem ao regime de alternância dos turnos têm sofrido com efeitos relacionados a esse sistema de horários, principalmente quando desempenham suas 
atividades de trabalho noturno. As principais alteraçóes encontradas referem-se à qualidade do sono, sonolência durante o turno, fadiga, estresse, alteraçôes de humor e diminuição no estado de alerta e vigilância. Esses sintomas estáo associados às alteraçôes do ritmo circadiano, as quais são causadas pelos horários irregulares dos turnos.

Além de prejuízos no desempenho das atividades referentes ao controle do tráfego aéreo, a irregularidade do sono é capaz de elevar o risco de erros nas tomadas de decisóes. O trabalho em turnos pode majorar a exposição ao adoecimento ao impor a irregularidade de horários, o que causa prejuízos no cotidiano relacionado aos laços sociofamiliares e ao engajamento do indivíduo em atividades singulares a si, fora de sua atividade laboral.

A caracterização dos conceitos relativos à terapia ocupacional nos últimos anos nos mostra as inúmeras possibilidades que os terapeutas ocupacionais têm diante de si. Magalhães (2013) demostra a extraordinária tarefa de maximizar as possibilidades ocupacionais de indivíduos e grupos com os quais trabalham, a partir do reconhecimento do enorme potencial da ocupação criativa, significativa e transformadora.

É necessário o desenvolvimento das investigaçóes relacionadas aos regimes de turnos que permitam o acompanhamento mais preciso da sua influência na saúde ocupacional dos CTA, principalmente em relação às questóes externas ao ambiente de trabalho, o quão eles afetam as situaçóes da vida social e a qualidade de vida desse profissional essencial para a segurança da aviação e campo primário da Terapia Ocupacional.

\section{Referências}

ALENCAR, M. C. B.; BIZ, R. A. M. Relaçóes entre condiçôes e organização do trabalho e os afastamentos de trabalhadores portuários de transporte. Revista de Terapia Ocupacional da Universidade de São Paulo, São Paulo, v. 23, n. 3, p. 208-215, 2012.

ARAÚJO, R. C. S. S. O trabalho na aviação e as práticas de saúde sob o olhar do controlador de tráfego aéreo. 2000. 118 f. Dissertação (Mestrado em Saúde do Trabalhador) - Universidade de São Paulo, São Paulo, 2000.

BISSERET, A. Analysis of mental processes involved in air traffic control. Ergonomics, Bethesda, v. 14, n. 5, p. 565-570, 1971. PMid:5148232

BRASIL. Ministério da Defesa. Portal da Força Aérea Brasileira. Disponível em: <http://www.fab.mil.br/index.php>. Acesso em: 15 jun. 2014.

CARUSO, C. C. et al. Overtime and extended work shifts: recent findings on illnesses, injuries, and health behaviors. Cincinnati: NIOSH Publications Dissemination, 2004.

CARVALHO, P. V.; FERREIRA, B. Modeling activities in air traffic control systems: antecedents and consequences of a mid-air collision. Work: A Journal of Prevention, Assessment, and Rehabilitation, Netherlands, v. 41, p. 232-239, 2012. Suplemento 1. PMid:22316728

CASTRO, E. D.; LIMA, E. M. F. A.; BRUNELlO, A. I. B. Atividades humanas e terapia ocupacional. In: DE CARLO, M. M. R. P.; BARTALOTTI, C. C. Terapia ocupacional no Brasil: fundamentos e perspectivas. São Paulo: Plexus, 2001. p. 41-59.

COSTA, G. Evaluation of workload in air traffic controllers. Ergonomics, Bethesda, v. 36, n. 9, p. 1111-1120, 1993. PMid:8404839

CRUZ, C. et al. Clockwise and counterclockwise rotating shifts: effects on sleep duration, timing, and quality. Aviation, Space, and Environmental Medicine, Alexandria, v. 74, n. 6 Pt 1, p. 597-605, 2003a. PMid:12793529.

CRUZ, C. et al. Clockwise and counterclockwise rotating shifts: effects on vigilance and performance. Aviation, Space, and Environmental Medicine, Alexandria, v. 74, n. 6 Pt 1, p. 606-614, 2003b. PMid:12793530.

DEPARTAMENTO DE CONTROLE DO ESPAÇO AÉREO - DECEA. Gerenciamento do tráfego aéreo. Disponível em: <http://www.decea.gov.br/espaco-aereo/ gerenciamento-de-trafego-aereo/>. Acesso em: 06 maio 2014.

EDWARDS, T. et al. Factor interaction influences on human performance in air traffic control: the need for a multifactorial model. Work : a Journal of Prevention, Assessment, and Rehabilitation, Netherlands, v. 41, n. 1, p. 159-166, 2012. Suplemento 1. PMid:22316716

EMMEL, M. L. G. Trabalho e qualidade de vida dos terapeutas ocupacionais: estudo de uma amostra brasileira. Cadernos de Terapia Ocupacional da UFSCar, São Carlos, v. 20 , n. 1, p. 55-63, 2012.

FISCHER, F. M. Relevância dos fatores psicossociais do trabalho na saúde do trabalhador. Revista de Saúde Pública, São Paulo, v. 46, n. 3, p. 401-406, 2012.http:// dx.doi.org/10.1590/S0034-89102012000300001. PMid:22635034

FISCHER, F. M.; LIEBER, R. R. Trabalho em turnos. In: MENDES, R. Patologias do trabalho. Rio de Janeiro: Atheneu, 2003. p. 825-868.

FLO, E. et al. Shift work disorder in nurses — assessment, prevalence and related health problems. PLOS ONE, San Francisco, v. 7, n. 4, p. e33981, 2012. PMid:22485153

FOLKARD, S.; CONDON, R. Night shift paralysis in air traffic control officers. Ergonomics, Bethesda, v. 30, n. 9, p. 1353-1363, 1987. PMid:3428257

FROST, P.; KOLSTAD, H. A.; BONDE, J. P. Shift work and the risk of ischemic heart disease - a systematic review of the epidemiologic evidence. Scandinavian Journal of Work, Environment \& Health, Helsinki, v. 35, 
n. 3, p. 163-179, 2009. Disponível em: <http://www. sjweh.fi/show_abstract.php?abstract_id=1319>. Acesso em: 22 maio 2014. PMid:19387517

JOU, R. C.; KUO, C. W.; TANG, M. L. A study of job stress and turnover tendency among air traffic controllers: the mediating effects of job satisfaction. Transportation Research Part E: Logistics and Transportation Review, Taiwan, v. 57, p. 95-104, 2013. Disponível em: <http:// isiarticles.com/bundles/Article/pre/pdf/6190.pdf> Acesso em: 20 maio 2014.

KIRCHNER, J. H.; LAURIG, W. The human operator in air traffic control systems. Ergonomics, Bethesda, v. 14, n. 5, p. 549-556, 1971. PMid:5148230

KOSTREVA, M.; MCNELIS, E.; CLEMENS, E. Using a circadian rhythms model to evaluate shift schedules. Ergonomics, Bethesda, v. 45, n. 11, p. 739-763, 2002. PMid: 12487689

KUO, C. W.; JOU, R. C.; LIN, S. W. Turnover intention of air traffic controllers in Taiwan: a note. Journal of Air Transport Management, London, v. 25, p. 50-52, 2012. Disponível em: <http://linkinghub.elsevier.com/ retrieve/pii/S096969971200107X>. Acesso em: 15 maio 2014.

LANCMAN, S.; GHIRARDI, M. I. G. Pensando novas práticas em terapia ocupacional, saúde e trabalho. Revista de Terapia Ocupacional da Universidade de São Paulo, São Paulo, v. 13, n. 2, p. 44-50, 2002.

LANCMAN, S. Saúde, trabalho e terapia ocupacional. São Paulo: Roca, 2004.

LIBERATI, A. et al. The PRISMA statement for reporting systematic reviews and meta-analyses of studies that evaluate health care interventions: explanation and elaboration. Annals of Internal Medicine, Philadelphia, v. 151, n. 4, p. W65-W94, 2009. Suplemento 1. PMid:19622512.

MAGALHĀES, L. Ocupação e atividade: tendências e tensóes conceituais na literatura anglófona da terapia ocupacional e da ciência ocupacional. Cadernos de Terapia Ocupacional da UFSCar, São Carlos, v. 21, n. 2, p. 255-263, 2013.

MIGUEL, N. B. et al. Avaliação ergonômica do trabalho do controlador de tráfego aéreo em uma torre de controle de aeródromo. In: ENCONTRO NACIONAL DE ENGENHARIA DE PRODUÇÃO, 26., 2006, Fortaleza. Anais... Fortaleza: ABEPRO, 2006. p. 1-7.

MOTTER, A. A.; GONTIJO, L. A. Análise da carga de trabalho em sistemas complexos: gestão da variabilidade e imprevisibilidade nas atividades do controlador de tráfego aéreo. 2007. 219 f. Tese (Doutorado em Engenharia de Produção) - Universidade Federal de Santa Catarina, Florianópolis, 2007.

NATALE, V.; MARTONI, M.; CICOGNA, P. Effects of circadian typology on sleep-wake behavior of air traffic controllers. Psychiatry and Clinical Neurosciences, Australia, v. 57, n. 5, p. 539-541, 2003. Suplemento 1.PMid: 12950710
NOONE, P. Shift work risk of stroke and metabolic syndrome. Occupational Medicine, Oxford, v. 59, n. 7, p. 521-521, 2009.Disponível em: <http://occmed.oxfordjournals.org/cgi/doi/10.1093/occmed/kqp107>. Acesso em: 15 maio. 2014.

PÁDULA, R. S. et al. Análise da apresentação textual de revisões sistemáticas em fisioterapia publicadas no idioma português. Revista Brasileira de Fisioterapia, São Carlos, v. 16, n. 4, p. 281-288, 2012. PMid:22858736.

PAN, A. et al. Rotating night shift work and risk of type 2 diabetes: two prospective cohort studies in women. PLOS Medicine, San Francisco, v. 8, n. 12, p. e1001141, 2011. PMid:22162955

PRUCHNICKI, S. A.; WU, L. J.; BELENKY, G. An exploration of the utility of mathematical modeling predicting fatigue from sleep/wake history and circadian phase applied in accident analysis and prevention: the crash of Comair Flight 5191. Accident Analysis and Prevention, Orlando, v. 43, n. 3, p. 1056-1061, 2011. PMid:21376901

SANTOS, C. M. C.; PIMENTA, C. B. A.; NOBRE, M. R. C. A estratégia PICO para a construção da pergunta de pesquisa e busca de evidências. Revista Latino-Americana de Enfermagem, Ribeirão Preto, v. 15, n. 3, p. 508-511, 2007.PMid:17653438.

SCHROEDER, D. J.; ROSA, R. R.; WITT, L. A. Some effects of 8-vs. 10-hour work schedules on the test performance/alertness of air traffic control specialists. International Journal of Industrial Ergonomics, Virginia, v. 21, p. 307-321, 1998. Disponível em: <http://www.sciencedirect.com/science/article/pii/S0169814197000449>. Acesso em: 09 jun. 2014.

SILVA, M. P. Sistemas de trabalho em turnos e seus impactos sobre a saúde dos trabalhadores: uma abordagem ergonômica. 2008. 108 f. Dissertação (Mestrado em Engenharia de Produção) - Universidade Federal do Rio Grande do Sul, Porto Alegre, 2008.

SIGNAL, T. L.; GANDER, P. H. Rapid counterclockwise shift rotation in air traffic control: effects on sleep and night work. Aviation, Space, and Environmental Medicine, Alexandria, v. 78, n. 9, p. 878-885, 2007. PMid:17891898

SOARES, C. P.; PINHEIRO, G. M.; SOUSA, P. J. S. A influência das variabilidades na carga de trabalho dos controladores de tráfego aéreo. In: ENCONTRO NACIONAL DE ENGENHARIA DE PRODUÇÃO, 31 ., 2011, Belo Horizonte. Anais... Belo Horizonte: ABEPRO, 2011. p. 1-13

SOUZA, S. B. et al. Influência do turno de trabalho e cronotipo na qualidade de vida dos trabalhadores de enfermagem. Revista Gaúcha de Enfermagem / EENFUFRGS, Porto Alegre, v. 33, n. 4, p. 79-85, 2012. PMid:23596920.

STOYNEV, A. G.; MINKOVA, N. K. Effect of forward rapidly rotating shift work on circadian rhythms of arte- 
rial pressure, heart rate and oral temperature in air traffic controllers. Occupational Medicine, Oxford, v. 48, n. 2, p. 75-79, 1998. PMid:9614764

\section{Contribuição dos Autores}

Alisson Vieira Marcolino: autor principal, realizou a concepção do texto, coleta dos dados, organização de fontes e análises, redação do texto e confecção da figura e tabela. Joseana Celiza Fernandes Siqueira: realizou a orientação e revisão do estudo. Bárbara Iansã de Lima Barros: colaborou com a concepção do texto e realizou a orientação e revisão em todas as etapas do estudo. Todos os autores aprovaram a versão final do texto. 